\title{
Notch3 Overexpression Is Related to the Recurrence of Ovarian Cancer and Confers Resistance to Carboplatin
}

\author{
Joon Tae Park, ${ }^{\star \dagger}$ Xu Chen, ${ }^{* \ddagger}$ \\ Claes G. Tropè, ${ }^{\S}$ ता Ben Davidson, ${ }^{\text {,III }}$ \\ le-Ming Shih, ${ }^{* \dagger}$ and Tian-Li Wang ${ }^{\ddagger}$ \\ From the Departments of Pathology," Pathobiology Graduate \\ Program, ${ }^{\dagger}$ and Oncology, Gynecology, and Obstetrics, Johns \\ Hopkins Medical Institutions, Baltimore, Maryland; the Section \\ for Gynecologic Oncology, Division of Gynecology and \\ Obstetrics, and the Division of Pathology, Norwegian Radium \\ Hospital, Oslo University Hospital, Oslo, Norway; and The \\ Medical Faculty, ${ }^{\text {Tा }}$ University of Oslo, Oslo, Norway
}

Amplification of the Notch3 locus has been detected in ovarian high-grade serous carcinoma (HGSC), the most common and malignant type of ovarian cancer. We have previously demonstrated that ovarian cancer cells, which amplified and overexpressed Notch3, were dependent on Notch3 signaling for cellular survival and growth. In this study, we provide new evidence that Notch 3 expression is associated with recurrent postchemotherapy HGSCs. Moreover, patients with recurrent HGSCs in effusion with high Notch3 expression had a significantly worse clinical outcome, including reduced overall survival and shortened progression-free survival than did patients with low Notch3 expressing HGSC. Ectopic expression of the Notch 3 intracellular domain led to an increase in $\mathrm{IC}_{50}$ for carboplatin in an ovarian surface epithelial cell line and in a low-grade serous carcinoma cell line that expressed undetectable levels of Notch3. Interestingly, expression of the Notch3 intracellular domain increased expression of several genes associated with embryonic stem cells including Nanog, Oct4, Klf4, Rex1, Rif1, Sall4, and $N A C 1$ as well as an ATP-dependent transporter gene, $A B C B 1$. Knockdown of Notch3 resulted in sensitization to carboplatin in OVCAR3 that expresses abundant Notch3. Taken together, the above findings suggest that Notch3 pathway activation reprograms tumor cells to assume an array of embryonic stem cell markers and participates in development of chemoresistance in HGSC. (Am J Pathol 2010, 177:1087-1094; DOI: 10.2353/ajpath.2010.100316)
It has been established that embryonic development and cancer pathogenesis share several molecular features including activation of pathways involving Hedgehog, Wnt, and Notch. In normal tissues, these pathways are important in governing tissue differentiation, cell renewal, and regeneration. Aberrations in homeostatic signaling pathways have been known to participate in the development of several types of human cancer. Ovarian carcinoma is the most lethal gynecologic cancer and is the fifth leading cause of cancer mortality in women. ${ }^{1}$ Over the past few years, we have focused on the molecular etiology of ovarian cancer using several genome-wide methods. Based on digital karyotyping and single nucleotide polymorphism arrays to detect DNA copy number changes in affinity-purified ovarian cancer cells from surgical specimens, we identified a novel amplicon located in the chromosome $19 p 13.12$ region in nearly 8 to $19 \%$ of ovarian high-grade serous carcinomas, the most common and lethal type of ovarian cancer. ${ }^{2}$ Comprehensive mRNA expression analysis of all genes within the minimal amplicon identified Notch3 as the gene that showed the most significant overexpression in amplified tumors compared with that in nonamplified tumors. Notch3 DNA copy number was positively correlated with Notch3 protein expression based on parallel immunohistochemical and fluorescence in situ hybridization studies. ${ }^{2}$ In addition, an independent study based on transcriptome analysis has confirmed that Notch3 is an up-regulated gene in ovarian cancer. ${ }^{3}$ The above reports indicate an important role for

Supported by the American Cancer Society (grant RSG-08-174-01GMC to T.-L.W.), the Ovarian Cancer Research Fund (T.-L.W.), a National Institutes of Health $(\mathrm{NIH})$ Specialized Program of Research Excellence (SPORE) Career Development Award (T.-L.W.), NIH National Cancer Institute (grants R01-CA103937 to I.-M.S. and R01-CA129080 to I.M.S.), and the Inger and Jon Fredriksen Foundation for Ovarian Cancer Research (B.D.).

Accepted for publication May 11, 2010.

Supplemental material for this article can be found on http://ajp. amjpathol.org

Address reprint requests to Tian-Li Wang, Ph.D., CRB-II, Room 306, 1550 Orleans St., Johns Hopkins Medical Institutions, Baltimore, MD 21231. E-mail: tlw@jhmi.edu. 
Notch3 in the development of ovarian high-grade serous carcinoma. Indeed, functional inactivation of Notch3 by either $\gamma$-secretase inhibitor or Notch3-specific small interfering RNA led to suppression of cell proliferation and induction of apoptosis in cell lines that overexpressed Notch3 but not in cell lines that expressed a minimal amount of Notch3. ${ }^{2}$

One of the critical challenges in the management of patients with ovarian cancer is the development of chemoresistant recurrent disease. Although platinum-based chemotherapy has improved the 5 -year survival rate, the overall mortality of ovarian cancer has remained largely unchanged because most patients eventually develop recurrent carcinomas after chemotherapy and eventually succumb to their disease. Thus, recurrent tumors represent the true "killer," as the primary ovarian tumors are removed by surgery. Until the mechanism of recurrence in ovarian cancer becomes more clearly understood, attempts to develop new therapies will remain empirical. ${ }^{4,5}$ In this study, we investigated the biological roles of Notch3 signaling in tumor progression by analyzing clinical samples to determine whether Notch3 pathway activation is related to tumor recurrence. Furthermore, because ovarian tumors often develop resistance to carboplatin, which is routinely used to treat patients with ovarian cancer, we modified Notch signaling activity in cell culture systems to assess possible contribution of the Notch3 pathway to carboplatin resistance.

\section{Materials and Methods}

\section{Reagents and Cell Lines}

The ovarian cancer cell line, OVCAR3, was purchased from American Type Culture Collection (Rockville, MD). The immortalized normal ovarian surface epithelial cell line, IOSE-80pc, was kindly provided by Dr. Nelly Auersperg (University of British Columbia, Vancouver, BC, Canada), and the micropapillary (low-grade) serous ovarian carcinoma cell line, MPSC1 was previously established by us. ${ }^{27}$ All cell lines used in this study were cultured in RPMI 1640 medium containing 5\% fetal bovine serum.

\section{Immunohistochemistry}

A rabbit polyclonal anti-Notch3 antibody (Santa Cruz Biotechnology, Santa Cruz, CA) ${ }^{2,6}$ was used in immunohistochemical analysis. Tissue microarrays containing 21 paired primary and recurrent ovarian serous carcinoma tissues were deparaffinized, and antigen was retrieved in a citrate buffer ( $\mathrm{pH}$ 6.0) for 10 minutes. Immunostaining was performed using an EnVision+ System peroxidase kit (Dako, Carpinteria, CA). Immunostaining intensity was scored as negative (0), negligible (1+), moderate (2+), or intense (3+); two investigators independently scored all of the samples. In discordant cases, a third investigator scored the samples, and the final intensity score was determined by majority vote.

\section{Effusion Tumor Samples}

Specimens were obtained from patients with either ovarian carcinoma (OC) $(n=65)$, primary peritoneal serous carcinoma $(n=11)$, or tubal serous carcinoma $(n=2)$. Because of their similar histogenesis, morphology, and treatment, in this report these are all referred to as OC. OC specimens and relevant clinical data were obtained from the Division of Gynecology and Obstetrics, Norwegian Radium Hospital, Oslo University Hospital (Table 1). Effusions were submitted for routine diagnostic purposes to the Division of Pathology, Norwegian Radium Hospital, Oslo University Hospital, during 1998 to 2005. Specimens were centrifuged, and pellets were fresh-frozen at $-70^{\circ} \mathrm{C}$ in RPMI 1640 medium supplemented with $25 \%$ fetal calf serum and 10\% dimethyl sulfoxide. Diagnoses were established using morphology and immunohistochemistry. ${ }^{28}$ The Regional Committee for Medical Research Ethics in Norway approved the study. Statistical analysis was performed using the SPSS-PC package (version 16, SPSS, Chicago, IL). Probabilities of $\leq 0.05$ were considered significant. Survival data were available for all patients. Clinicopathologic parameters were grouped as follows: age, $\leq 60$ versus $>60$ years; grade, 1 to 2 versus 3; International Federation of Gynecology and Obstetrics (FIGO) stage, III versus IV; and response to chemotherapy, complete versus partial response/stable disease/progression/allergic or adverse reaction. Analysis of the association with response to chemother-

Table 1. Clinicopathologic Data of Patients with Ovarian Carcinoma $(n=78)$

\begin{tabular}{|c|c|c|}
\hline Parameter & $\begin{array}{c}\text { Primary } \\
\text { diagnosis } \\
(n=36)\end{array}$ & $\begin{array}{l}\text { Disease } \\
\text { recurrence } \\
(n=42)\end{array}$ \\
\hline Age (mean; range) & $61 ; 38-79$ & $60 ; 34-83$ \\
\hline \multicolumn{3}{|l|}{ FIGO stage } \\
\hline II & 1 & 1 \\
\hline III & 18 & 25 \\
\hline IV & 17 & 15 \\
\hline NA & 0 & 1 \\
\hline \multicolumn{3}{|l|}{ Grade } \\
\hline Low & 3 & 3 \\
\hline High* & 27 & 36 \\
\hline $\mathrm{NA}^{\dagger}$ & 6 & 3 \\
\hline \multicolumn{3}{|l|}{ Histology } \\
\hline Serous & 28 & 37 \\
\hline Mucinous & 1 & 0 \\
\hline Clear cell & 1 & 1 \\
\hline Endometrioid & 0 & 1 \\
\hline Undifferentiated & 1 & 1 \\
\hline Other $r^{\ddagger}$ & 4 & 2 \\
\hline$N A^{\S}$ & 1 & 0 \\
\hline \multicolumn{3}{|l|}{ Residual disease } \\
\hline$\leq 1 \mathrm{~cm}$ & 17 & 14 \\
\hline$>1 \mathrm{~cm}$ & 14 & 23 \\
\hline $\mathrm{NA}^{\dagger}$ & 5 & 5 \\
\hline
\end{tabular}

NA, not available.

*Including 2 patients with clear cell carcinoma.

${ }^{+}$Including 7 effusions from inoperable patients for which the biopsy sample was too small for grading and 2 patients with no data.

FIncluding mixed epithelial tumors and carcinomas not otherwise specified.

§Patient with inoperable disease, whose biopsy sample had too little tissue to establish histological type. 
apy for primary disease was limited to primary diagnosis specimens. Univariate survival analyses of overall survival (OS) and progression-free survival (PFS) were performed using the Kaplan-Meier method and log-rank test. For these analyses, Notch3 expression was grouped as low versus high based on median values. Multivariate survival analysis was performed using the Cox proportional hazards model.

\section{Production of NICD3-V5 Retrovirus}

The PCR product of the Notch3 intracellular domain (NICD3; amino acids 1663 to 2312) was cloned into an expression vector, pcDNA6, with a V5 tag. The coding sequence of NICD3-V5 was subcloned into the pBabepuro retroviral vector, and high-titer retroviral stocks were generated using a Phoenix ecotropic packaging cell line. Retroviral gene transfer was performed as described previously. ${ }^{29}$ Cells infected with the empty pBabe-puro retroviral vector were used as controls.

\section{Western Blot Analysis}

Protein lysates were prepared by resuspending cell pellets in Laemmli sample buffer containing $5 \% \beta$-mercaptoethanol. Protein lysates were separated by 4 to $12 \%$ Tris-glycine gel electrophoresis and transferred onto a polyvinylidene difluoride membrane using a semidry apparatus (Bio-Rad Laboratories, Hercules, CA). The membrane was blocked with 5\% nonfat dry milk in TBST (20 $\mathrm{mmol} / \mathrm{L}$ Tris- $\mathrm{HCl}, 0.5 \mathrm{M} \mathrm{NaCl}$, and $0.1 \%$ Tween 20), incubated with anti-Notch3 antibody (Santa Cruz Biotechnology) ${ }^{2,6}$ or anti-V5 antibody (Invitrogen, Carlsbad, CA) at room temperature for 3 hours, followed by washing with TBST. Subsequently the membrane was incubated with horseradish peroxidase-conjugated secondary antibody and detected with ECL solution (Thermo Fisher Scientific, Waltham, MA).

\section{Drug Resistance Assay}

Cells were seeded in 96-well plates at a density of 3000 cells/well. After overnight culture, the cells were treated with serial concentrations of carboplatin. After an additional 48 to 96 hours, cell number was measured by the incorporation of SYBR Green I nucleic acid gel stain (Molecular Probes, Eugene, OR) using a fluorescence microplate reader (FLUOstar, BMG, Durham, NC). Data were determined from six replicates and are expressed as the fold increase relative to the control group. $\mathrm{IC}_{50}$ was defined as the concentration that resulted in a $50 \%$ decrease in the number of cells compared with control cultures in the absence of the drug.

\section{Notch3 Short Hairpin RNA Transduction}

A Notch3 short hairpin RNA (shRNA) plasmid that targeted the Notch3 coding sequence (5'-CTCGGTAGTAATGCTGGAGAT-3') was purchased from the Mission
shRNA collection of Sigma-Aldrich (St. Louis, MO). The shRNA sequence was subcloned into a pLKO.1-puro vector. Viral supernatants were generated by transfecting 293-T cells with the shRNA construct and packaging vectors pSPAX2 and pMD2.G (Addgene, Cambridge, $M A)$. The high-titer lentiviral supernatants in the presence of $8 \mu \mathrm{g} / \mathrm{ml}$ Polybrene were used to infect target cells.

\section{Quantitative Real-Time PCR}

Relative transcript expression levels were measured by quantitative real-time PCR using a method described previously. ${ }^{30}$ The primer sequences are shown in Table 2. PCR reactions were performed in triplicate using an iCycler (Bio-Rad Laboratories). The amplified products were quantified by fluorescence intensity of SYBR Green I (Molecular Probes). Average fold changes were calculated by differences in threshold cycles $\left(C_{t}\right)$ between pairs of samples to be compared. The amyloid beta (A4) precursor protein $(A P P)$ was used for normalizing the cDNA concentration of each sample.

\section{Results}

\section{Notch3 Pathway Activation in Recurrent Ovarian Cancer}

To determine whether Notch3 pathway activation was related to tumor progression, we used immunohistochemical analysis to analyze the expression levels of Notch3 in tumor cells. In particular, we focused on the immunostaining intensity of the intracellular domain (NICD3) in nuclei, because NICD3 has been established as a surrogate marker for Notch pathway activation. The specificity of the anti-Notch3 antibody was demonstrated in our previous studies. ${ }^{2,6}$ A total of 21 pairs of primary and matched recurrent ovarian high-grade serous carcinomas were studied. The results demonstrated that NICD3 immunoreactivity was significantly elevated in the nuclei of recurrent ovarian serous carcinoma tissues compared with primary carcinoma tissues from the same patients (Figure 1A). Among 21 patients, 15 showed increased NICD3 immunostaining intensity in recurrent tumors compared with their corresponding primary tumors, 3 showed no difference, and 3 showed a decrease in recurrent comparing to primary tumors. Fisher's exact analysis demonstrated a statistically significant elevation in NICD3 in recurrent compared with primary carcinomas $(P<0.001)$. Representative paired primary and recurrent ovarian serous carcinoma tissues are shown in Figure 1B.

\section{Notch3 Activation Is Associated with Poor Prognosis in Recurrent Postchemotherapy Ovarian Cancer}

Based on the above findings, we sought to investigate whether Notch3 expression was associated with clinical outcomes including OS and PFS. A cohort of 78 patients 
Table 2. Primer Sequences for Quantitative PCR Analysis

\begin{tabular}{|c|c|c|}
\hline Gene symbol & Forward primer & Reverse primer \\
\hline NANOG & 5'-AAAGCTTGCCTTGCTTTGAA-3' & 5'-AAGTGGGTTGTTTGCCTTTG-3' \\
\hline OCT4 & 5'-GCCATACGGTCACAGAGCTT-3' & 5'-GGAAGCTTAGCCAGGTCAGA-3' \\
\hline$R E X 1$ & 5'-GACAGACTGACCCTAAAGCAAGACGA-3' & 5'-GGTATCCGTCAGGGAAGCCATCT-3' \\
\hline$A B C B 1$ & $5^{\prime}-$ TCACTTCAGGAAGCAACCAG- $3^{\prime}$ & $5^{\prime}-$ ATTCCTCGAGAAACTGCGAA-3' \\
\hline RIF1 & 5'-TACGGAGGGTCTTCCTGAAA-3' & 5'-TCCTTGGGCACCTTTATCTG-3' \\
\hline KLF4 & $5^{\prime}$-AGAGTTCCCATCTCAAGGCA-3' & 5'-GTCAGTTCATCTGAGCGGG-3' \\
\hline SALL4 & $5^{\prime}$-ACCCCGGAGTTTGCAGAT-3' & 5'-СTTCATCCTCACTCGCCAC-3' \\
\hline NAC1 & $5^{\prime}-$ CCAGACACTGCAGATGGAGA-3' & 5'-AAGCTGAGGATCTGCTGGAA-3' \\
\hline ITGB1 & $5^{\prime}-$ TGTATACAAGCAGGGCCAAA- $^{\prime}$ & 5'-TTCCTTTGCTACGGTTGGTT-3' \\
\hline SOX2 & 5'-AACCCCAAGATGCACAACTC-3' & 5'-GCTTAGCCTCGTCGATGAAC- $3^{\prime}$ \\
\hline$D A X 1$ & $5^{\prime}$-AGTACGCCTACCTCAAGGGG-3' & 5'-CTGAGTTCCCCACTGGAGTC-3' \\
\hline CD44 & $5^{\prime}$-CCACGTGGAGAAAAATGGTC- $3^{\prime}$ & $5^{\prime}-$ TACACCCCTGTGTTGTTTGC- $3^{\prime}$ \\
\hline NOTCH3 & $5^{\prime}$-GTGTGTGTCAATGGCTGGAC-3' & $5^{\prime}$-GTGACACAGGAGGCCAGTCT-3' \\
\hline$P B X 1$ & $5^{\prime}$-CGGAGCTGGAGAAATACGAG-3' & 5'-CTGTCGCTTGCTTGTTGAAA-3' \\
\hline HES1 & 5'-CGGACATTCTGGAAATGACA-3' & 5'-GTGCGCACCTCGGTATTAAC-3' \\
\hline HEY2 & $5^{\prime}$-CGTCGGGATCGGATAAATAA-3' & 5'-GCACTCTCGGAATCCTATGC-3' \\
\hline HEYI & $5^{\prime}$-GATGCAAGCCAGGAAGAAAC-3' & 5'-GGCATGGAGCATTTTCAAGT-3' \\
\hline TLE2 & $5^{\prime}$-AGGGTCATCCGTCTCTTCCT-3' & $5^{\prime}-\mathrm{ACACCTTCACACAGCCCTTG-3'}$ \\
\hline TLE3 & $5^{\prime}$-AAATTCACGGTGGCTGAGTC-3' & $5^{\prime}$-TTCAAGCCATAGGACATCTCA-3' \\
\hline TLE4 & $5^{\prime}-$ TGAGAAACTCGCCAGTGAGA-3' & $5^{\prime}$-CTGGAGTTGTTGCCCAATG-3' \\
\hline TLE6 & 5'-CTGCATGTGAAGGAGCAGTT-3' & 5'-TTGTAGCCCTTGACCACGAT-3' \\
\hline HERPUD1 & 5'-TTGGACCTATTCAGCAGCTACA-3' & $5^{\prime}-$ TCAGGAGGAGGACCATCATT-3' \\
\hline MYC & $5^{\prime}-$ ССТАСССТCTCAACGACAGC-3' & $5^{\prime}$-СTCTGACCTTTTGCCAGGAG-3' \\
\hline$A P P$ & 5'-TGAATAATTGGGTTTGTTTTGT-3' & 5'-ТАСААССТАССТТСТСТСССТT-3' \\
\hline
\end{tabular}

who presented with ovarian carcinoma, predominantly of the high-grade serous type, in effusions were analyzed using quantitative real-time PCR. All patients had tumor spread beyond the ovary, the majority diagnosed as FIGO stage III to IV. Of the patients, 75 received chemotherapy at diagnosis, of whom 68 were treated with carboplatin (53 combined with paclitaxel, 6 combined with paclitaxel and gemcitabine, and 9 as a single agent). A summary of the clinical data of the patients is shown in

\section{A}

Notch3 Expression in Paired Primary/Recurrent Tumors

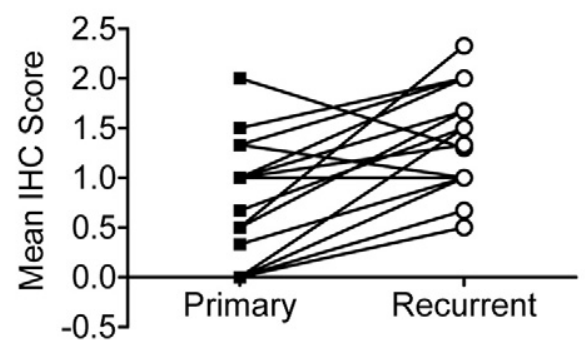

B

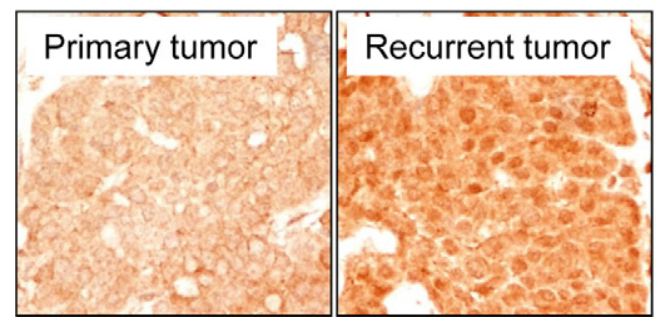

Figure 1. Notch3 protein is overexpressed in recurrent ovarian carcinoma. A: Immunostaining of 21 pairs of primary and recurrent tumors from the same patients using a Notch3-specific antibody. B: Representative paired primary and recurrent ovarian serous carcinoma tissues are shown. Nuclear Notch3 staining was observed in recurrent ovarian carcinoma tissues.
Table 1. An arbitrary cutoff based on the median relative Notch3 transcript level was used to identify specimens as either high Notch3 transcript expression or low Notch3 transcript expression. Among the 78 patients, 36 were women with primary diagnosis specimens obtained before chemotherapy administration and 42 were women with recurrent tumors after chemotherapy. Kaplan-Meier analysis was performed in these two groups (primary versus recurrent). The results demonstrated that expression levels of Notch3 were not associated with disease outcome in primary diagnosis effusions $(P>0.05$, data not shown). However, within the recurrent tumor group, patients with high Notch3 expression showed a statistically significant worse overall and progression-free survival than did patients with low Notch3 expression. Patients with high Notch3 expression $(n=13)$ had a mean OS time of 22 months versus 37 months for patients with low Notch3 expression ( $n=29, P=0.002$ ) (Figure 2A). Patients with high Notch3 expression $(n=12)$ had a mean PFS of 3 months versus 8 months for patients with low Notch3 expression ( $n=29$; $P=0.042$; one patient with no PFS data) (Figure 2B).

The clinical parameters that significantly correlated with $O S$ in univariate analysis of patients with disease recurrence effusions were FIGO stage $(P=0.018)$ and response to chemotherapy at first disease recurrence $(P=0.02)$. In Cox multivariate analysis, Notch levels $(P=$ $0.005)$, FIGO stage $(P=0.03)$, and response to chemotherapy at first disease recurrence $(P=0.034)$ were all independent prognostic factors. The only clinical parameter that significantly correlated with PFS in univariate analysis was FIGO stage $(P<0.001)$. Response to chemotherapy at diagnosis $(P=0.053)$ was additionally entered into the Cox analysis. In Cox multivariate analysis, only FIGO stage $(P=0.002)$ was an independent 
A

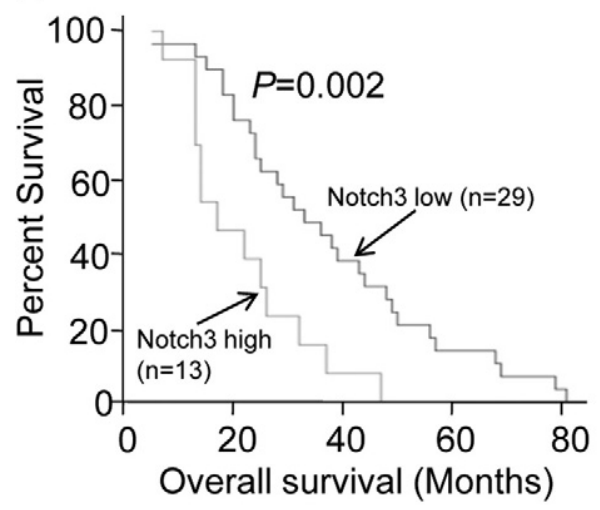

B

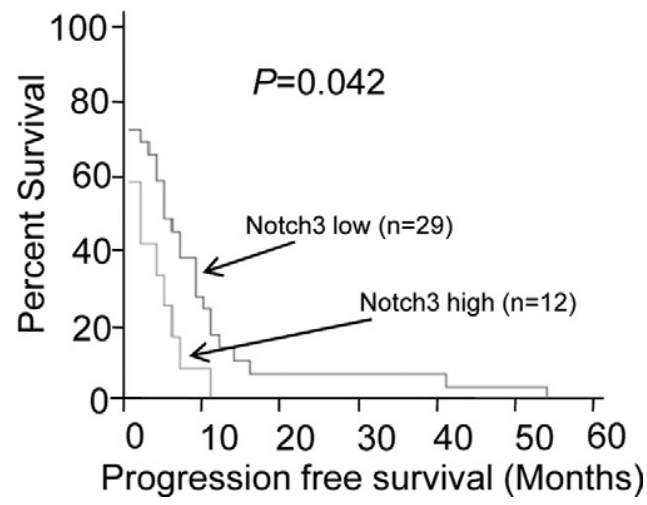

Figure 2. Notch3 expression correlates with poor survival in ovarian carcinoma. A: Kaplan-Meier survival curve shows an association between Notch3 mRNA expression and OS for 42 patients with recurrent (postchemotherapy) effusions. Patients with high Notch 3 expression (based on median cutoff) ( $n=13$, gray line) had a mean OS of 22 months versus 37 months for patients whose effusions showed low Notch3 expression ( $n=29$, black line; $P=$ 0.002). B: Kaplan-Meier survival curve shows the association between Notch3 mRNA expression and PFS for 42 patients with recurrent (postchemotherapy) effusions. Patients with high Notch3 expression (based on median cutoff) ( $n=12$, gray line) had a mean PFS of three months versus eight months for patients whose effusions showed low Notch3 expression ( $n=29$, black line; $P=0.042$ ). One patient had no PFS data.

predictor, with a marginal value $(P=0.05)$ for Notch mRNA levels.

\section{Notch3 Expression and Carboplatin Resistance}

Because development of drug resistance is a major factor for tumor recurrence, we next examined whether ectopic expression of Notch3 in a cell culture model would affect the sensitivity of cells to carboplatin. IOSE-80pc, an immortalized ovarian surface epithelial cell line, and MPSC1, a low-grade ovarian serous tumor cell line, were transduced with retrovirus containing NICD3 or with the empty vector control. The cell lines were chosen because of their low endogenous level of Notch3. The ectopic expression of NICD3 was confirmed by Western blot analysis (Figure 3). Both cell lines with engineered NICD3 expression were found to be more resistant to carboplatin than control virus-infected cells (Figure $3 \mathrm{~A}$ ). The $I \mathrm{C}_{50}$ values for carboplatin were 35 and $15 \mu \mathrm{mol} / \mathrm{L}$ in NICD3infected IOSE-80pc and MPSC1 and were 3.0 and 5.8 $\mu \mathrm{mol} / \mathrm{L}$ in control virus-infected IOSE-80pc and MPSC1, respectively (Figure $3 \mathrm{~A}$ ).

The above results suggested that NICD3 was sufficient to confer carboplatin resistance. To determine whether Notch3 expression was required for carboplatin resistance, we knocked down Notch3 in an ovarian cancer cell line (OVCAR3) that expresses abundant Notch3. Lentivirus carrying the Notch3 shRNA was used to transduce OVCAR3, and the efficiency of Notch3 knockdown was demonstrated by Western blot analysis (Figure 3B, top panel). OVCAR3 cells in the Notch3 shRNA-treated group were found to be somewhat more sensitive to carboplatin than cells in the control shRNA-treated group; the IC50 of carboplatin was $60 \mu \mathrm{mol} / \mathrm{L}$ in the Notch3 shRNA-treated group compared with $80 \mu \mathrm{mol} / \mathrm{L}$ in the control group (Figure 3B, bottom panel).

\section{Ectopic Expression of Notch3 Increased the Expression of Embryonic Stem Cell Markers}

To explore possible mechanisms involved in carboplatin resistance mediated by Notch3, we used a candidate approach to identify potential genes whose expression was regulated by Notch3 signaling. Because stem cell phenotypes may contribute to the development of drug resistance, we selected those genes that have been identified as embryonic stem cell markers including NANOG, OCT4, REX1, CD44, ITGB1, NAC1, SOX2, KLF4, SALL4, and RIF1. To test whether overexpression of Notch3 up-regulated embryonic stem cell markers, we performed quantitative real-time PCR in both IOSE-80pc and MPSC1 cell lines. We found that NICD3 virus-transduced cells had increased expression levels of many of the genes tested. Most remarkably, NICD3 enhanced the expression of NANOG, OCT4, REX1, RIF1, and SALL4 in both IOSE-80pc and MPSC1 cell lines (Figure 4). The effect of NICD3 ectopic expression on well-known Notch target genes is shown in Supplemental Figure 1 (see http://ajp.amjpathol.org). The transcript levels of Hes1, Hey2, TLE2, and Pbx1 were significantly increased in NICD3-transduced IOSE-80pc and MPSC1 cells.

\section{Discussion}

In most patients, ovarian carcinoma is diagnosed at an advanced stage and is treated with platinum-based chemotherapy after cytoreduction surgery. Unfortunately, acquisition of a chemoresistant phenotype in tumors is almost inevitable and ultimately leads to clinically incurable disease. Understanding and targeting the molecular underpinnings of recurrent disease continues to be the principal challenge in cancer research. ${ }^{7}$ In this study we provide evidence that Notch3 signaling participates in the development of recurrent ovarian serous carcinoma probably through enhancing carboplatin resistance in tumor cells. Given the multifaceted role of the Notch pathway in development of cancer, our findings should have several important implications. 

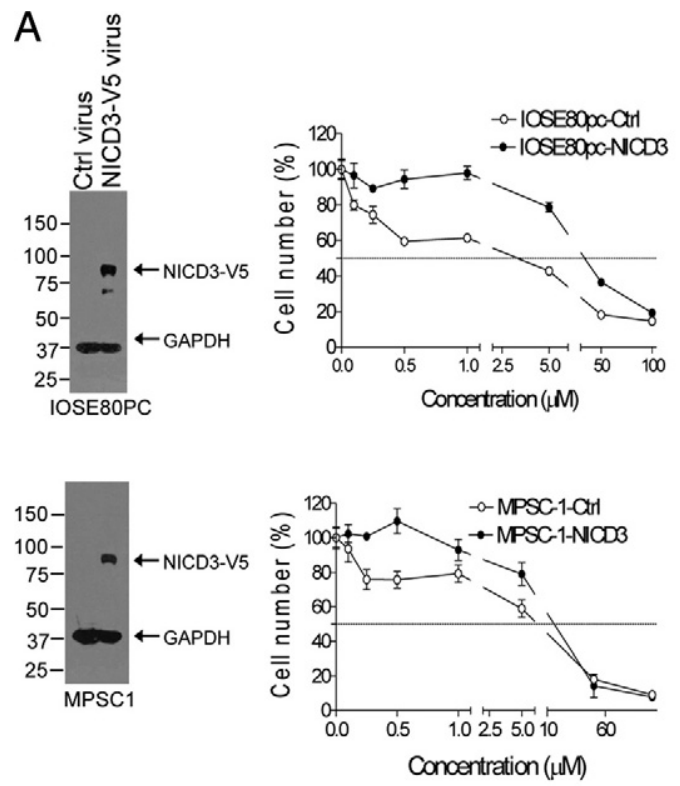
comes from our observations that Notch3 overexpression and the presence of nuclear NICD3 were more frequently seen in recurrent postchemotherapy ovarian serous carcinomas than in their primary counterparts. This finding suggests that activation of the Notch3 signaling pathway is advantageous for cancer cell survival under the selection pressure of chemotherapy. This view of positive selection is supported by our in vitro study showing that NICD3 expression is sufficient to confer carboplatin resistance in two different cell lines. Moreover, this study
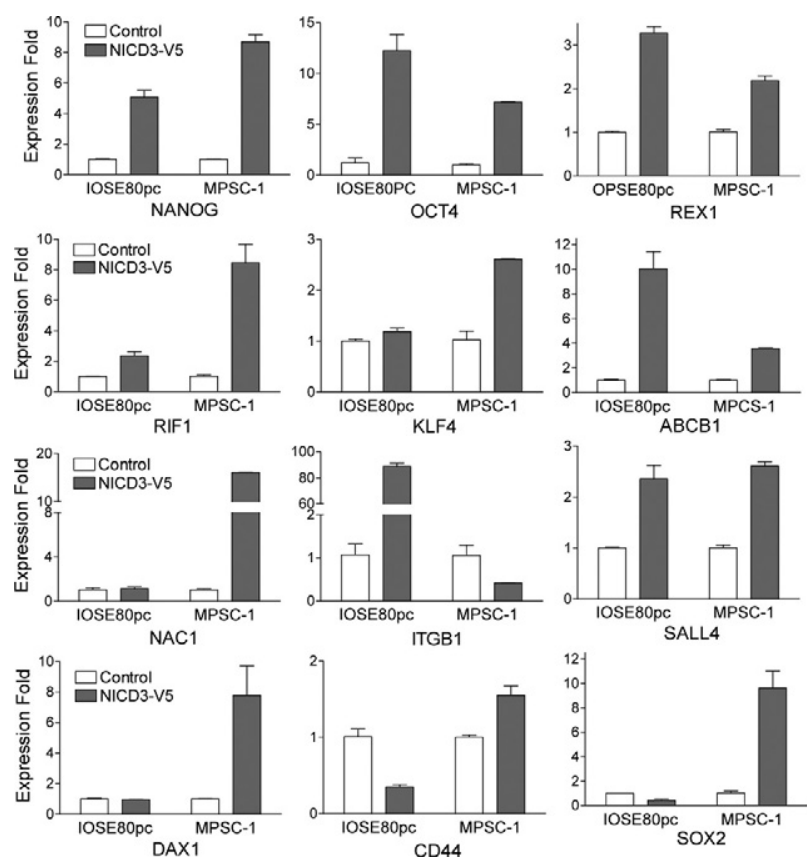

Figure 4. Ectopic expression of Notch3 enhanced the expression of stem cell markers. Quantitative real-time PCR was performed on previously identified stem cell markers in NICD3-V5 virus-infected versus control virusinfected cells
Figure 3. NICD3 expression is sufficient and essential to induce carboplatin resistance. A: IOSE-80pc and MPSC1 cells were transduced with the NICD3-V5 retrovirus and stained with anti-V5 antibody in Western blot analysis. A single protein band, corresponding to the molecular mass of NICD3 protein is shown in NICD3 transduced cells but not in control virus-transduced cells. Both NICD3-expressing IOSE-80pc and MPSC1 cells show increased resistance to carboplatin compared with the control virus-infected cells. B: Notch3 shRNA transduction in OVCAR3 significantly reduces Notch3 protein expression compared with the control shRNA transduction and mock control (top panel). Notch3 shRNA treatment sensitizes ovarian cancer cells toward carboplatin as evidenced by an reduction in $\mathrm{IC}_{50}$ (bottom panel). identified up-regulation of ABCB1 on ectopic Notch3 expression. $A B C B 1$ is a member of the superfamily of ATPbinding cassette $(A B C)$ transporters. More specifically, it belongs to the multiple drug resistance (MDR) protein subfamily, members of which are known to participate in multidrug resistance through an ATP-dependent drug efflux pumping mechanism for xenobiotic compounds with broad substrate specificity. ${ }^{8}$ As with other MDR proteins, ABCB1 up-regulation by Notch3 signaling may be responsible for decreased drug accumulation in cancer cells and mediate the development of carboplatin resistance.

In contrast to the significant shift of $\mathrm{IC}_{50}$ in the forward approach (ectopic expression of NICD3), there was only a subtle decrease in $\mathrm{IC}_{50}$ when Notch3 expression was knocked down in OVCAR3 cells. Although this finding suggests that Notch3 is required to maintain carboplatin resistance, our result underscores the fact that multiple genes and pathways contribute to drug resistance in ovarian cancer cells. To this end, we have reported that expression levels of NAC1, Rsf-1 (HBXAP), fatty acid synthase, and annexin A11 were significantly higher in recurrent, high-grade ovarian serous carcinoma specimens after chemotherapy, and, more importantly, expression of these genes played a causal role in conferring drug resistance in vitro. ${ }^{9-14}$ Furthermore, our recent proteomic analysis identified RELA and STAT5 as two major proteins associated with carboplatin resistance in ovarian tumors. ${ }^{31}$ We showed that both RELA and STAT5 bound to the $\mathrm{BCl}-\mathrm{xL}$ promoter and regulated its expression. This finding is biologically significant in light of the fact that $\mathrm{Bcl}-\mathrm{xL}$ has been identified as one of the key regulators for cell survival in the presence of cytotoxic reagents. ${ }^{15,16}$ Thus, it could be envisioned that the Notch3 pathway may collaborate with other molecules in the development of drug resistance in ovarian high-grade serous carcinoma. 
The finding that there was a significant correlation between a higher level of Notch3 mRNA expression and worse overall and progression-free survival in recurrent ovarian cancer effusions is highly interesting. However, a similar correlation was not observed when primary diagnosis effusions were analyzed. This is not surprising because cancer cells obtained from peritoneal or pleural fluid (effusion) at diagnosis have been shown to differ in their biological nature from those obtained at disease recurrence, and expression of cancer-associated molecules in these two specimen types has previously demonstrated to exhibit different prognostic roles. ${ }^{17-20}$

Although the utility of Notch3 expression as a prognostic marker awaits further confirmation in independent studies, our results indicate that Notch3 could be a therapeutic target for the most malignant forms and advanced stages of ovarian serous carcinoma, ie, recurrent tumors in effusion. Similar to targeting the hedgehog pathway, targeting the Notch pathway has recently become a subject of intense research for cancer therapeutics because mounting evidence from preclinical studies has demonstrated that $\gamma$-secretase inhibitors hold promise as a new target-based therapy for those tumors with Notch activation. $\gamma$-Secretase inhibitors prevent cleavage of the oncogenic (intracellular) domain of Notch molecules, ie, NICD, and suppress Notch activity. ${ }^{21}$ Despite several challenges on the way, it is expected that new generations of specific $\gamma$-secretase inhibitors will be tested in patients with ovarian cancer who have developed carboplatin-resistant diseases.

The current study also provides new evidence that Notch3 could function to orchestrate a "stem cell-like" property in ovarian cancer, as suggested by the fact that ectopic NICD3 expression induced expression of several embryonic stem cell markers. Many of these proteins have been demonstrated to form a protein interaction network that is critical to establish and/or maintain embryonic cell pluripotency. ${ }^{22,23}$ Because the "stemness" phenotype may be involved in generating chemoresistance, ${ }^{24,25}$ our findings point toward the possibility that Notch3 signaling is related to drug resistance and therefore tumor recurrence. For example, Nanog, a homeobox-containing transcription factor, is required to maintain pluripotency in mouse embryonic stem cells. ${ }^{26}$ It has been reported that Nanog stimulated Stat3 transcriptional activation, MDR1 overexpression, and multidrug resistance. Conversely, down-regulation or inactivation of Nanog signaling not only suppressed HA/CD44-mediated tumor cell behaviors but also increased chemosensitivity. ${ }^{25}$

In summary, our data show that Notch3 signaling may participate in tumor recurrence by conferring cancer cells with a drug-resistant phenotype. Overexpression of Notch3 in cancer cells is associated with the most aggressive phenotype, and Notch3 signaling may turn out to be a prognostic marker in ovarian high-grade serous carcinoma. Future studies should focus on investigating the biological significance of and links among Notch pathway activation, stem cell phenotypes, and chemoresistance. We believe that this study represents an initial but fundamental step toward elucidating the molecular pathogenesis of aggressive behavior associated with high-grade serous carcinoma and that our findings will inspire future investigations in developing Notch3-based therapy to combat drug resistance in ovarian cancer.

\section{References}

1. Jemal A, Siegel R, Ward E, Hao Y, Xu J, Thun MJ: Cancer statistics, 2009. CA Cancer J Clin 2009, 59:225-249

2. Park JT, Li M, Nakayama N, Davidson B, Eberhart CG, Kurman RJ Shih I-M, Wang T-L: Notch-3 gene amplification in ovarian cancer. Cancer Res 2006, 66:6312-6318

3. Lu KH, Patterson AP, Wang L, Marquez RT, Atkinson EN, Baggerly KA, Ramoth LR, Rosen DG, Liu J, Hellstrom I, Smith D, Hartmann L, Fishman D, Berchuck A, Schmandt R, Whitaker R, Gershenson DM, Mills GB, Bast RC Jr: Selection of potential markers for epithelial ovarian cancer with gene expression arrays and recursive descent partition analysis. Clin Cancer Res 2004, 10:3291-3300

4. Dubeau L: Ovarian cancer. The Metabolic and Molecular Basis of Inherited Disease, ed 8. Edited by CR Scriver, AL Beaudet, WS Sly, D Valle, B Childs, KW Kinzler, B Vogelstein: New York, McGraw-Hill, 2001, pp 1091-1096

5. Berek JS, Martinez-Maza O: Molecular and biologic factors in the pathogenesis of ovarian cancer. J Reprod Med 1994, 39:241-248

6. Park JT, Shih le M, Wang TL: Identification of pbx1, a potential oncogene, as a notch3 target gene in ovarian cancer. Cancer Res 2008, 68:8852-8860

7. Cho KR, Shih IM: Ovarian cancer. Annu Rev Pathol Mech Dis 2009 $4: 287-313$

8. Higgins CF: Multiple molecular mechanisms for multidrug resistance transporters. Nature 2007, 446:749-757

9. Shih le M, Sheu JJ, Santillan A, Nakayama K, Yen MJ, Bristow RE, Vang R, Parmigiani G, Kurman RJ, Trope CG, Davidson B, Wang TL: Amplification of a chromatin remodeling gene, Rsf-1/HBXAP, in ovarian carcinoma. Proc Natl Acad Sci USA 2005, 102:14004-14009

10. Choi JH, Sheu JJ, Guan B, Jinawath N, Markowski P, Wang TL, Shih le M: Functional analysis of 11 q13.5 amplicon identifies Rsf-1 (HBXAP) as a gene involved in paclitaxel resistance in ovarian cancer. Cancer Res 2009, 69:1407-1415

11. Nakayama K, Nakayama N, Davidson B, Sheu JJ, Jinawath N, Santillan A, Salani R, Bristow RE, Morin PJ, Kurman RJ, Wang TL, Shih le $\mathrm{M}$ : A BTB/POZ protein, NAC-1, is related to tumor recurrence and is essential for tumor growth and survival. Proc Natl Acad Sci USA 2006, 103:18739-18744

12. Jinawath N, Vasoontara C, Yap KL, Thiaville MM, Nakayama K, Wang TL, Shih IM: NAC-1, a potential stem cell pluripotency factor, contributes to paclitaxel resistance in ovarian cancer through inactivating Gadd45 pathway. Oncogene 2009, 28:1941-1948

13. Song J, Shih le M, Chan DW, Zhang Z: Suppression of annexin A11 in ovarian cancer: implications in chemoresistance. Neoplasia 2009, 11:605-614, 1 p following 614

14. Song J, Shih le M, Salani R, Chan DW, Zhang Z: Annexin XI is associated with cisplatin resistance and related to tumor recurrence in ovarian cancer patients. Clin Cancer Res 2007, 13:6842-6849

15. Williams J, Lucas PC, Griffith KA, Choi M, Fogoros S, Hu YY, Liu JR: Expression of $\mathrm{Bcl}-\mathrm{xL}$ in ovarian carcinoma is associated with chemoresistance and recurrent disease. Gynecol Oncol 2005, 96:287-295

16. Villedieu M, Louis MH, Dutoit S, Brotin E, Lincet $H$, Duigou F, Staedel C, Gauduchon P, Poulain L: Absence of Bcl-xL down-regulation in response to cisplatin is associated with chemoresistance in ovarian carcinoma cells. Gynecol Oncol 2007, 105:31-44

17. Kleinberg L, Florenes VA, Silins I, Haug K, Trope CG, Nesland JM, Davidson B: Nuclear expression of survivin is associated with improved survival in metastatic ovarian carcinoma. Cancer 2007, 109:228-238

18. Elloul S, Elstrand MB, Nesland JM, Trope CG, Kvalheim G, Goldberg I, Reich R, Davidson B: Snail, Slug, and Smad-interacting protein 1 as novel parameters of disease aggressiveness in metastatic ovarian and breast carcinoma. Cancer 2005, 103:1631-1643

19. Davidson B, Trope CG, Wang TL, Shih le M: Expression of the chromatin remodeling factor Rsf-1 is upregulated in ovarian carci- 
noma effusions and predicts poor survival. Gynecol Oncol 2006, 103:814-819

20. Davidson B, Alejandro E, Florenes VA, Goderstad JM, Risberg B, Kristensen GB, Trope CG, Kohn EC: Granulin-epithelin precursor is a novel prognostic marker in epithelial ovarian carcinoma. Cancer 2004, 100:2139-2147

21. Shih IM, Wang TL: Notch signaling, gamma-secretase inhibitors, and cancer therapy. Cancer Res 2007, 67:1879-1882

22. Wang JLD, Orkin SH: Requirement of Nanog dimerization for stem cell self-renewal and pluripotency. Proc Natl Acad Sci USA 2008, 105:6326-6331

23. Wang JRS, Chu J, Shen X, Levasseur DN, Theunissen TW, Orkin SH: A protein interaction network for pluripotency of embryonic stem cells. Nature 2006, 444:364-368

24. Gu G, Yuan J, Wills M, Kasper S: Prostate cancer cells with stem cell characteristics reconstitute the original human tumor in vivo. Cancer Res 2007, 67:4807-4815

25. Bourguignon LY, Peyrollier K, Xia W, Gilad E: Hyaluronan-CD44 interaction activates stem cell marker Nanog, Stat-3-mediated MDR1 gene expression, and ankyrin-regulated multidrug efflux in breast and ovarian tumor cells. J Biol Chem 2008, 283:17635-17651

26. Mitsui KTY, Itoh H, Segawa K, Murakami M, Takahashi K, Maruyama
M, Maeda M, Yamanaka S: The homeoprotein Nanog is required for maintenance of pluripotency in mouse epiblast and ES cells. Cell 2003, 113:631-642

27. Pohl G, Ho CL, Kurman RJ, Bristow R, Wang TL, Shih le M: Inactivation of the mitogen-activated protein kinase pathway as a potential target-based therapy in ovarian serous tumors with KRAS or BRAF mutations. Cancer Res 2005, 65:1994-2000

28. Davidson B: Ovarian carcinoma and serous effusions. Changing views regarding tumor progression and review of current literature. Anal Cell Pathol 2001, 23:107-128

29. Dang L, Fan X, Chaudhry A, Wang M, Gaiano N, Eberhart CG: Notch3 signaling initiates choroid plexus tumor formation. Oncogene 2006, 25:487-491

30. Buckhaults P, Zhang Z, Chen YC, Wang TL, St Croix B, Saha S, Bardelli A, Morin PJ, Polyak K, Hruban RH, Velculescu VE, Shih le M: Identifying tumor origin using a gene expression-based classification map. Cancer Res 2003, 63:4144-4149

31. Jinawath N, Vasoontara C, Jinawath A, Fang X, Zhao K, Yap KL, Guo T, Lee CS, Wang W, Balgley BM, Davidson B, Wang TL, Shih IM: Oncoproteomic analysis reveals coupregulation of RELA and STAT5 in carboplatin resistant ovarian carcinoma. PLoS ONE, 5:e11198, 2010. PMID: 20585448 